\title{
GLAD!
}

Revue sur le langage, le genre, les sexualités

$01 \mid 2016$

Varia

\section{Idéologies de genre et construction des savoirs en sciences phonétiques}

Gender Ideologies and the Construction of Knowledges in Phonetic Sciences

\section{Aron Arnold}

\section{(2) OpenEdition}

\section{Journals}

Édition électronique

URL : http://journals.openedition.org/glad/117

DOI : $10.4000 /$ glad. 117

ISSN : 2551-0819

Éditeur

Association GSL

\section{Référence électronique}

Aron Arnold, « Idéologies de genre et construction des savoirs en sciences phonétiques », GLAD! [En ligne], 01 | 2016, mis en ligne le 15 novembre 2016, consulté le 17 décembre 2020. URL : http:// journals.openedition.org/glad/117 ; DOI : https://doi.org/10.4000/glad.117

Ce document a été généré automatiquement le 17 décembre 2020.

\section{(c) (i) (9)}

La revue GLAD! est mise à disposition selon les termes de la Licence Creative Commons Attribution Pas d'Utilisation Commerciale - Pas de Modification 4.0 International. 


\title{
Idéologies de genre et construction des savoirs en sciences phonétiques
}

\author{
Gender Ideologies and the Construction of Knowledges in Phonetic Sciences
}

\author{
Aron Arnold
}

1 L'objectif de cet article est d'exposer comment les savoirs produits dans les sciences phonétiques sont, malgré leur objectivité et neutralité axiologique postulées, empreints d'idéologies de genre et contribuent à véhiculer celles-ci.

2 La notion d'idéologie de genre peut être définie comme «the set of beliefs that govern people's participation in the gender order, and by which they explain and justify that participation $^{1} »$ (Eckert \& McConnel-Ginet 2003: 35).

3 L'analyse d'un ensemble de travaux en sciences phonétiques a permis de constater que, de la même manière que la médecine a utilisé systématiquement le corps masculin pour représenter l'être humain, la littérature phonétique utilise systématiquement des conduits vocaux masculins pour représenter les conduits vocaux humains. Le corps masculin est ainsi érigé en norme. Cela a comme conséquence de traiter le corps féminin comme corps différent, comme corps spécifique, comme corps qui dévie de la norme. Depuis l'Antiquité, la médecine décrit le corps féminin comme inférieur à celui de l'homme, instable, pathologique. Une telle pathologisation peut également être retrouvée dans les études phonétiques portant sur le syndrome vocal prémenstruel. Finalement, on peut constater que la littérature phonétique véhicule une idéologie de genre binaire qui normalise les schémas femme/féminine/femelle et homme/ masculin/mâle.

4 Cet article vise à déconstruire l'idée que la production de savoirs est un acte neutre et objectif. Il s'agit de révéler le « caractère socialement et historiquement construit et genré » (Bourcier \& Moliner 2012: 87) de tout savoir et de l'idée même d'objectivité. Comme le rappelle Bourcier (Bourcier \& Moliner $2012: 89$ ), «l'objectivité scientifique, telle qu'elle a été construite et revendiquée, est un savoir situé et partiel. S'il ne se présente pas comme tel, c'est pour mieux se revendiquer comme neutre, universel ou transcendant ». 


\section{Le corps masculin comme norme}

5 Dans les études phonétiques, le corps est omniprésent. Dans les domaines de la phonétique articulatoire, acoustique ou clinique, il est schématisé, modélisé, représenté à travers une "iconographie dans laquelle la parole et l'émission d'un son sont imbriquées dans le corps d'un locuteur type » (Greco 2013 : 272). En phonétique, comme par ailleurs dans la majorité des disciplines scientifiques qui étudient l'anatomie et la physiologie du corps humain (la médecine, la biologie, etc.), le corps de ce locuteur type est un corps masculin blanc, figé, décontextualisé de tout environnement social. Dans ce cadre, l'utilisation du corps masculin comme corps type a pour effet d'instaurer ce corps en tant que norme, en tant qu'élément non marqué et d'instaurer le corps féminin comme élément spécifique, marqué.

\section{Le féminin comme Autre}

6 Dans la littérature scientifique, le corps féminin est régulièrement décrit à travers des comparaisons avec le corps masculin. En comparant le corps féminin au corps masculin - au corps «normal»- le corps féminin est présenté comme spécifique, voire anormal.

7 Des représentations du corps féminin comme corps masculin imparfait, qui ont perduré de l'Antiquité jusqu'aux Lumières, le corps féminin est devenu le corps différent à partir du XvIII ${ }^{e}$ siècle (Laqueur 1990). À une vision hiérarchique, dans laquelle le corps féminin n'était qu'un corps masculin «inversé » (le vagin étant p. ex. décrit comme un pénis inversé et les ovaires, des testicules inversés), s'est substituée une vision dans laquelle le corps féminin est perçu comme fondamentalement, essentiellement différent du corps masculin (et non l'inverse - il s'agit bien du corps féminin qui est différent du corps masculin et non pas du corps masculin qui est différent du corps féminin ${ }^{2}$ ). On est ainsi passé d'une vision unisexuelle du corps, véhiculée notamment par Galien, à une vision dimorphe, binaire, dans laquelle les hommes et les femmes sont décrits par le biais du contraste. Cette seconde vision n'a toutefois pas abandonné les fondements de la première : le corps féminin reste imparfait, faible, instable et sujet à toutes sortes de pathologies.

8 Lawrence et Bendixen (1992) ont montré que les représentations du corps féminin dans les manuels anatomiques publiés entre 1890-1989 suivaient régulièrement les conventions suivantes :

Choosing male illustrations for non-sex specific features, organizing chapters with "the [male]" headings distinct from "the female" sections, using explicit or implicit directional comparisons of female to male structures, placing female terms in parentheses, and directing readers to visualize female regions as altered male ones, all maintain an anatomical hierarchy: male, then female; male as norm, female as different ${ }^{3}$. (Lawrence \& Bendixen 1992 : 933)

On retrouve ces discours qui produisent le corps masculin en norme également dans la littérature phonétique, notamment dans l'utilisation systématique du conduit vocal masculin pour décrire, schématiser et modéliser le conduit vocal humain. 


\section{Confusion entre conduit vocal masculin et conduit vocal humain}

10 Le conduit vocal est le tube qui va de la glotte jusqu'aux lèvres. Dans la littérature, il est régulièrement décrit comme ayant une longueur de $17 \mathrm{~cm}$ ou $17,5 \mathrm{~cm}$ (p. ex. par Boë et al. 2007 ; Ormezzano 2000 : 131; Schroeder 2013: 6; Titze 2001). Cette longueur de $17 / 17,5 \mathrm{~cm}$ n'est cependant pas une moyenne des conduits vocaux humains, mais une moyenne des conduits vocaux masculins.

Figure : Illustration représentant le conduit vocal



site internet du Skidmore College 4

11 La confusion entre conduit vocal masculin et conduit vocal humain semble trouver son origine dans les premières publications de Fant datant des années 1960. Dans Acoustic Theory of Speech Production (1960), Fant utilise un modèle de conduit vocal de $17 \mathrm{~cm}$ en se basant sur des mesures tomographiques: (1) des clichés radiologiques du conduit vocal d'un locuteur russe de 38 ans (1960: 93) que Fant a réalisés lui-même dans le cadre de sa collaboration avec Roman Jakobson et Morris Halle au MIT (Fant 2004), ainsi que (2) des clichés qui avaient été réalisés précédemment par le radiologiste Paul Edholhm (1960 : 98).

Dans Preliminaries to Speech Analysis (1963), publié trois ans plus tard, Jakobson, Fant et Halle expliquent que le conduit vocal masculin a une longueur moyenne de $17,5 \mathrm{~cm}$. Puis, dans l'article "A note on vocal tract size factors and non-uniform F-pattern scalings » (1966), Fant réutilise les données de Edholm pour expliquer les différences acoustiques entre voyelles produites par des femmes et voyelles produites par des hommes par des différences entre conduits vocaux féminins et conduits vocaux masculins. Fant écrit que selon ces clichés, les hommes ont des cavités pharyngales moyennes de $9,1 \mathrm{~cm}$ et des cavités buccales moyennes de $8,25 \mathrm{~cm}$. Le conduit vocal 
masculin moyen mesuré par Fant avait ainsi une longueur totale de $17,35 \mathrm{~cm}$, qui sera ensuite arrondie à $17,5 \mathrm{~cm}$.

Ces trois travaux ont été cités dans d'innombrables publications phonétiques au cours des décennies suivantes et, de citation en citation, le conduit vocal masculin de 17/17,5 cm s'est transformé en « le conduit vocal humain ».

En étudiant la littérature phonétique, on peut constater que dans la majorité des publications, le conduit de 17/17,5 cm est (1) présenté comme « le conduit vocal », sans que l'auteur-e précise qu'il s'agit d'un conduit vocal masculin, ou (2) présenté en mentionnant qu'il s'agit d'un conduit vocal masculin, sans pour autant que l'auteur-e présente de conduit vocal féminin.

Voici quatre exemples de textes présentant un conduit vocal masculin comme «le conduit vocal » ou comme exemple d'un conduit vocal normal, typique :

(1) Thus, with an average vocal tract length about half the size $(8 \mathrm{~cm})$ of an adult human $(17 \mathrm{~cm})$, an infant a few months old produces vowels whose formants are two times greater than those produced by an adult ${ }^{5}$. (Boë et al. 2007 : 571)

(2) Toute fréquence de longueur d'onde inférieure à la longueur d'un résonateur, ici le conduit vocal (17,5 cm environ), va être modifiée par lui [...] (Ormezzano $2000: 131$ )

(3) [...] his resonance frequencies being noticably lower than those produced by a normal vocal tract of $170 \mathrm{~mm} \mathrm{length}^{6}$. (Schroeder 2013: 6)

(4) Figure 3 shows vocal tract inertance as a function of frequency for $\underline{\text { a }}$ $17.5 \mathrm{~cm}$ long vocal tract fully open at the mouth ${ }^{7}$. (Titze $2001: 523$ )

Le premier passage compare la longueur du conduit vocal du nourrisson à celui de l'adulte. Le conduit vocal « of an adult human » est décrit comme faisant $17 \mathrm{~cm}$. Dans le deuxième passage, qui est une description des propriétés acoustiques du conduit vocal, on peut lire que « le conduit vocal » a une longueur d'environ $17,5 \mathrm{~cm}$. L'article indéfini «an» dans «an adult human » et l'article défini «le» dans «le conduit vocal» ont dans ces deux cas la fonction de conférer un sens générique aux énoncés : un adulte humain, ça a un conduit vocal de $17 \mathrm{~cm}$; le conduit vocal, ça a une longueur de $17,5 \mathrm{~cm}$. Les deux énoncés impliquent que les conduits vocaux qui n'ont pas cette longueur ne sont pas des conduits vocaux habituels, typiques ou normaux. Dans le troisième passage, le conduit vocal de $170 \mathrm{~mm}$ est qualifié de «normal » (« a normal vocal tract of $170 \mathrm{~mm}$ length»). Décrire un conduit vocal de $170 \mathrm{~mm}$, donc un conduit vocal masculin, comme « conduit vocal normal » équivaut à dire que le corps masculin est le corps normal. Le quatrième passage est un exemple qui illustre la régularité à travers laquelle le conduit vocal masculin est utilisé dans des modélisations du conduit vocal humain. Que ce soient des modèles à un tube, à deux tubes, à trois tubes ou à $\mathrm{n}$ tubes (voir p. ex. Martin 2008: 127-141), on retrouve régulièrement des longueurs de conduits de $17 / 17,5 \mathrm{~cm}$.

17 Certains auteurs, comme p. ex. Ladefoged (1996), prétendent que cette longueur est utilisée afin de simplifier les calculs des résonances: « We will take the vocal tract to be a tube about $17.5 \mathrm{~cm}$ long (it is actually slightly less for most speakers, but this is a convenient figure because it keeps the arithmetic simple) $)^{8}$.» (Ladefoged $\left.1996: 116\right)$. S'il est vrai que des calculs avec le nombre 17,5 sont moins complexes que des calculs avec des nombres comprenant plusieurs décimales ou qui ne sont pas des multiples de 0,5, l'utilisation de ce nombre n'est cependant pas un choix anodin. D'autres mesures 
auraient tout aussi bien pu être utilisées dans des modélisations du conduit vocal. Mais c'est la mesure qui est associée au conduit vocal masculin qui est choisie.

Ces différents passages montrent donc comment le conduit vocal masculin est dégenré et universalisé et comment, par différentes stratégies discursives, il est transformé en conduit vocal humain.

19 Ci-après, on trouvera des exemples de textes qui décrivent un conduit vocal de $17 / 17,5 \mathrm{~cm}$ en précisant qu'il s'agit d'un conduit vocal masculin, sans pour autant présenter de conduit vocal féminin :

(1) An adult male vocal tract is approximately $17 \mathrm{~cm}$ long9. (Campbell, Jr. 1997 : 1441)

(2) Pour un tube de 17,5 centimètres, (longueur moyenne d'une bouche d'homme) fermé à un bout (la glotte) et ouvert à l'autre (les lèvres), il se produit des résonances à $500 \mathrm{cps}$ (premier formant), $1500 \mathrm{cps}$ (deuxième formant), $2500 \mathrm{cps}$ (troisième formant), $3500 \mathrm{cps}$ (quatrième formant), etc., qui correspondent au quart d'onde, aux trois-quarts d'onde, aux cinq-quarts d'onde, etc. (Delattre $1968: 62$ )

(3) Since the average length of the vocal tract of males is about $17.5 \mathrm{~cm}$, the resonances appear at approximately 500, 1500, 2500 cps. etc. ${ }^{10}$ (Jakobson, Fant \& Halle $1963: 18)$

(4) Chez les hommes adultes, la longueur du canal vocal est en moyenne de $17 \mathrm{~cm}$. (Landercy \& Renard $1979: 81$ )

20 Ces exemples montrent que les conduits vocaux féminins sont régulièrement ignorés. Cette omission contribue à la normalisation du corps masculin : on décrit le corps masculin parce que c'est lui qui est le corps de référence, le corps normal, le corps nonmarqué, c'est lui qui peut illustrer le corps humain alors que le corps féminin, toujours spécifique, toujours marqué, ne le pourrait pas.

21 Quand toutefois, des conduits vocaux féminins sont décrits dans la littérature phonétique, ils sont présentés après les conduits vocaux masculins et comparés à ceuxci pour expliquer à quel point ils sont différents de ces derniers. Par exemple, on explique que les cavités des femmes sont plus courtes que celles des hommes, plus étroites, etc.

(1) Un conduit vocal féminin est en moyenne $15 \%$ plus court qu'un conduit vocal masculin typique. En première approximation, la théorie acoustique indique que l'augmentation des fréquences de résonance est proportionnelle à la diminution de la longueur. Pour une forme constante, les formants devraient être $15 \%$ plus élevés chez les femmes que chez les hommes. (Calliope 1989: 86)

(2) This explains why adult female formant frequencies are higher than men on average, since a typical adult female vocal tract is shorter than that of an adult male ${ }^{11}$. (Dellwo, Huckvale, \& Ashby $2007: 16$ )

(3) A typical female vocal tract is 15-20 per cent shorter than that of a typical male $^{12}$. (Miranda $2012: 133$ )

(4) Some hypotheses for male-female differences in VOT have been stated based on the smaller vocal tract volume among women ${ }^{13}$. (Morris, McCrea, \& Herring $2008: 309$ )

Ces différents passages prennent comme référence le corps masculin et comparent le corps féminin avec celui-ci. Les quatre premiers passages présentent le conduit vocal féminin comme plus court que le conduit vocal masculin ( $15 \%$ plus court qu'un 
conduit vocal masculin typique "; «shorter than that of an adult male»; "15-20 per cent shorter than that of a typical male "; "the smaller vocal tract volume among women ») et le deuxième passage explique que les fréquences de résonance des femmes sont plus élevées que celles des hommes ( are higher than men on average »). Dans ces passages, le corps féminin est toujours comparé, jamais comparant.

Ceci illustre parfaitement ce que Guillaumin a expliqué dans « Pratique du pouvoir et idée de Nature » (Guillaumin 1978) :

Nous [les femmes] sommes toujours "plus" ou "moins". Et jamais nous ne sommes le terme de référence. On ne mesure pas la taille des hommes par rapport à la nôtre alors qu'on mesure la nôtre par rapport à celle des hommes [...], laquelle n'est mesurée que par rapport à elle-même. (Guillaumin 1978 : 16)

Ainsi, la manière de présenter et de différencier les conduits vocaux féminins des conduits vocaux masculins véhicule un discours qui produit le masculin comme norme et le féminin comme spécifique et, conséquemment, produit une opposition humain/ femme. Ceci fait écho aux travaux de Michard sur le genre en français contemporain. Elle explique que le masculin et le féminin n'ont pas la même fonction :

L'opposition fondamentale entre propriétés définissantes des notions d'homme et de femme, dont je fais l'hypothèse qu'elle correspond à l'opposition des signifiés du genre, est par conséquent : humain/femelle et le genre dit masculin est toujours générique du point de vue du sens humain, quelle que soit son extension, tandis que le féminin ne l'est jamais. (Michard $2000: 142$ )

Il est également intéressant de noter que certains ouvrages phonétiques vont jusqu'à consacrer un chapitre entier aux femmes alors que dans les autres chapitres, homme et humain sont confondus. Par exemple, Ormezzano (2000) dédie un chapitre entier à « La femme et ses hormones " dans lequel il décrit des spécificités des voix de femmes et leur lien avec les hormones. Dans ce chapitre, il explique que «la femme est sous l'influence de deux types d'hormones sexuelles " (2000: 207), les estrogènes et la progestérone. L'homme quant à lui n'est jamais décrit comme étant "sous l'influence » d'hormones.

L'instauration du corps masculin en tant que norme a par ailleurs comme effet que davantage d'études phonétiques sont faites sur des locuteurs masculins que sur des locuteurs féminins. Henton (1986) a analysé 42 études phonétiques censées présenter des données acoustiques représentatives de locuteurs adultes. Elle a remarqué que $30,9 \%$ de ces études ont été réalisées sur un nombre égal de locuteurs féminins et masculins, $40,5 \%$ ont été réalisées uniquement sur des locuteurs masculins, et seulement $4,8 \%$ ont été réalisées uniquement sur des locuteurs féminins. Parmi les études réalisées sur des locuteurs féminins et masculins, 21,4\% comptaient plus de locuteurs masculins que de locuteurs féminins; une seule étude comportait plus de locuteurs féminins.

\section{Le corps féminin pathologisé}

Une des conséquences de la constitution du corps masculin en norme est la pathologisation du corps féminin. Celle-ci a commencé dès les débuts de la médecine et perdure jusqu'à aujourd'hui. Ces discours pathologisant le corps féminin peuvent être retrouvés dans la littérature sur le syndrome prémenstruel et le syndrome vocal prémenstruel. 


\section{Pathologisation des changements cycliques féminins}

Selon Dorlin, c'est « [d]epuis Hippocrate et son traité des Maladies des femmes, [que l']on sait que les médecins considèrent que le corps féminin est particulièrement pathogène» (Dorlin 2005 : 135). Pour Hippocrate, ce sont les déplacements de l'utérus dans le corps des femmes en fonction du cycle lunaire qui provoquent leurs troubles physiques et mentaux (Rodin 1992). Décrit comme froid et humide, le corps féminin est, selon Aristote, incapable de produire une chaleur suffisante pour transformer le sang en semence (Dorlin 2006 ; Héritier 1984). Selon Galien, pour qui le corps féminin diffère de celui de l'homme par le fait que les organes génitaux soient "replié[s] en dedans ", c'est aussi à cause de l'incapacité des femmes de produire de la chaleur que leurs organes génitaux ne peuvent « descendre et faire saillie au-dehors» (Dorlin 2006:21). La froideur et l'humidité, associées à un tempérament flegmatique, rendent les femmes " "naturellement" plus faibles parce qu'elles sont physiquement imparfaites, empêchées par un corps constamment malade » (Dorlin 2006 : 24).

Comme il a été expliqué plus haut, cette conception du corps féminin comme version imparfaite et instable du corps masculin perdure jusqu'au $\mathrm{XVIII}^{\mathrm{e}}$ siècle pour céder sa place à une rhétorique de la différence : le corps féminin est à partir de ce moment décrit comme fondamentalement différent du corps masculin. C'est ce changement de paradigme, le passage d'un modèle à un sexe (one-sex model) à un modèle à deux sexes (two-sex model), qui a fait que les organes génitaux féminins et masculins sont perçus comme fondamentalement différents, incomparables (Laqueur 1990).

Un reflet de cette différenciation peut être trouvé dans l'intégration de nouveaux mots dans le lexique médical pour désigner des parties du corps féminin et masculin. Par exemple, Laqueur indique que «[f]or two millennia the ovary, an organ that by the early nineteenth century had become a synecdoche for woman, had not even a name of its own ${ }^{14}$ » (1990: 4). Avant le passage au modèle à deux sexes, un même terme était utilisé pour désigner les ovaires et les testicules. Chez Galien, en fonction du contexte, le terme " orcheis » pouvait faire référence aux gonades féminines ou masculines. De même, chez Hérophile, le terme « didymoi » (signifiant « jumeaux » en grec) désignait aussi bien les ovaires que les testicules (Laqueur 1990 : 4).

31 En s'appuyant sur cette différence anatomique, la médecine a construit l'idée de pathologies typiquement féminines, comme l'hystérie ${ }^{15}$, et ainsi l'image d'une femme régie par les hormones, les fluides corporels, etc., naturellement/biologiquement inférieure à l'homme.

\section{Du syndrome prémenstruel au syndrome vocal prémenstruel}

32 Je prendrai comme exemple de cette pathologisation du corps féminin le syndrome prémenstruel et le syndrome vocal prémenstruel. Le terme « syndrome prémenstruel » est apparu dans la littérature médicale dans les années 1950 et a depuis été utilisé pour 
désigner un ensemble de phénomènes qui ont lieu durant la phase lutéale - c'est-àdire la période entre l'ovulation et le dernier jour du cycle menstruel. Depuis l'apparition du terme "syndrome prémenstruel» ces phénomènes cycliques sont régulièrement décrits en termes de symptômes. Le « syndrome prémenstruel » est ainsi une version moderne des discours qui constituent le corps féminin en corps pathologiquement instable.

L'idée de syndrome prémenstruel trouve ses origines dans les études sur les «tensions prémenstruelles » qui ont été initiées par Frank dans les années 1930. L'objectif de ces études était d'établir des relations entre des changements hormonaux pendant le cycle menstruel et certaines manifestations psychologiques comme la dépression et l'irritabilité (Frank 1931). Dans la lignée de ces travaux se trouve l'article «The Premenstrual Syndrome » (1953) dans lequel Greene et Dalton introduisent le terme de "syndrome prémenstruel» pour désigner approximativement 150 symptômes qui surviendraient cycliquement et qui seraient dus aux fluctuations hormonales accompagnant les différentes phases des cycles menstruels.

Comme l'indique Kendall (1991), l'étape finale du processus de médicalisation, l'institutionnalisation du syndrome prémenstruel, a présentement été atteinte par son inclusion dans le DSM ${ }^{16} \mathrm{IV}$, publié en 1994. Les termes «syndrome prémenstruel » et «trouble dysphorique prémenstruel » y recouvrent un large spectre de phénomènes présentés comme dysfonctionnements physiologiques et psychologiques.

Il est cependant intéressant de noter, d'une part, qu'une grande partie de ces phénomènes ne sont pas uniquement observés chez des femmes, mais peuvent aussi être observés chez des hommes ainsi que chez des femmes ménopausées et, d'autre part, que dans des cultures non occidentales, ces phénomènes ne sont pas systématiquement associés aux cycles menstruels (Johnson 1987). Comme l'indique par ailleurs Zita (1988), il ne s'agit pas de nier qu'il existe des phénomènes corporels qui accompagnent les cycles menstruels, mais de montrer comment la littérature médicale pathologise ces phénomènes cycliques en les désignant avec des termes comme " symptôme » et « syndrome ".

Parallèlement aux études sur le syndrome prémenstruel a été construite l'idée de syndrome vocal prémenstruel. Par le terme de «syndrome vocal prémenstruel » sont désignés un ensemble de phénomènes vocaux liés à la cyclicité féminine. En comparant les données issues d'un ensemble d'études, Behr Davis et Lee Davis (1993) expliquent que l'on cite comme principaux symptômes du syndrome vocal prémenstruel un enrouement, des hémorragies laryngées, des troubles de la trachée, des raideurs des articulations laryngées, des œdèmes et rougeurs des plis vocaux. Ces symptômes provoqueraient ensuite des problèmes de hauteur vocale, d'intensité, de qualité de voix, voire une aphonie totale.

Dans la littérature sur le syndrome vocal prémenstruel, on peut retrouver la mobilisation d'un argumentaire similaire à celui que l'on retrouve, d'une part, dans des textes médicaux qui présentent le corps féminin comme fragile, instable et contrôlé par les hormones, et d'autre part, dans la littérature sur le syndrome prémenstruel, où des phénomènes cycliques sont décrits en termes de symptômes.

(1) In 38 women, we found 22 vocal premenstrual syndromes, presenting with hoarse voice and an increase of voice fatigue. Of these 22 women, all had luteal insufficiency as confirmed by vocal cord smear. Sixteen women did not have any particular voice change during the premenstrual phase, 
although two of them had luteal insufficiency. Fourteen had normal hormonal secretion ${ }^{17}$. (Abitbol et al. 1989)

(2) Premenstrual vocal syndrome is characterized by vocal fatigue, decreased range, a loss of power and loss of certain harmonics. The syndrome usually starts some 4-5 days before menstruation in some $33 \%$ of women. Vocal professionals are particularly affected. Dynamic vocal exploration by televideoendoscopy shows congestion, microvarices, edema of the posterior third of the vocal folds and a loss of its vibratory amplitude ${ }^{18}$. (Abitbol, Abitbol, \& Abitbol 1999 : 424)

(3) La voix blessée par les hormones (Abitbol 2005 [2013: 234]); Les cordes vocales bougent normalement, mais si la gauche est d'aspect normal, la corde droite est effectivement malade [...] (id. : 235) ; L'équilibre précaire de cet instrument exceptionnel [le larynx féminin] vient d'être blessé. (ibid. : 236) ; Nous sommes en période prémenstruelle, elle est fragilisée. (ibid.: 237) ; Chez toutes les femmes, on constate un syndrome prémenstruel. Mais seulement un tiers d'entre elles présente un œdème plus sévère des cordes vocales et un gonflement des seins témoin de l'équilibre précaire des hormones sexuelles. (ibid. : 238)

(4) The purpose of this paper is to present hoarseness as an unrecognized symptom of premenstrual tension, briefly to describe this entity, to discuss the mechanism accounting for vocal huskiness and loss of vocal control, and to give clinical examples ${ }^{19}$. (Frable $1962: 66$ ) fragilité et l'instabilité (p. ex. « insuffisance », " perte de force », « fatigue », « blessée », " équilibre précaire ", "fragilisée », " loss of vocal control », etc.), présentent une certaine ressemblance avec les descriptions du corps féminin comme version instable et imparfaite du corps masculin évoquées précédemment. Le troisième extrait est issu d'une section intitulée « La voix blessée par les hormones » du livre L'odyssée de la voix de Abitbol (2013 [2005] : 234). Dans cette section, l'auteur relate sa prise en charge d'une chanteuse lyrique dont la "voix vient de se blesser " à cause des hormones et explique que chez « toutes les femmes, on constate un syndrome prémenstruel » (id.).

Si Abitbol constate un syndrome prémenstruel chez "toutes les femmes», il est cependant important de noter que d'autres auteur-e-s n'ont trouvé aucune différence significative entre locutrices en période prémenstruelle et locutrices hors période prémenstruelle, ou encore entre locutrices en période prémenstruelle et locuteurs masculins. Par exemple, Meurer et al. (2009) ont enregistré 23 adolescentes pendant les phases folliculaire et lutéale de leurs cycles menstruels et n'ont trouvé aucune différence d'intensité, de hauteur vocale, de timbre ou encore de débit. Des résultats similaires ont été obtenus par Silverman et Zimmer (1978). Une première série d'enregistrements de 20 étudiantes n'a pas permis de trouver de différences de raucité de voix entre période prémenstruelle et période d'ovulation. L'expérience a été répliquée une deuxième fois avec 27 étudiantes et les résultats étaient identiques. Une autre étude portant sur les perturbations vocales durant le cycle menstruel a été réalisée par Higgins et Saxman (1989). Cette étude se démarque des autres par le fait qu'elle a été réalisée en se basant non seulement sur des analyses d'enregistrements de jeunes femmes, mais aussi d'enregistrements de jeunes hommes. Dix femmes et cinq hommes ont été enregistrés sur une période de 33 jours et des analyses acoustiques ont ensuite été effectuées pour calculer le jitter (variation de fréquence) dans leur voix. Higgins et Saxman n'ont pas trouvé de différences significatives entre la période prémenstruelle et le début de la menstruation (phase folliculaire) chez la majorité des 
participantes, mais notent un pic de perturbations au moment de l'ovulation. Ce qui est particulièrement intéressant est que, sur la période des 33 jours, de plus grandes perturbations ont été remarquées chez les hommes que chez les femmes.

Les études sur le syndrome prémenstruel et les études sur le syndrome vocal prémenstruel illustrent comment le fait d'avoir érigé le corps masculin en norme aboutit à une pathologisation de la cyclicité et, de cette manière, du corps féminin. Parce que le corps masculin - corps qui est considéré comme stable et n'étant soumis à aucune variation cyclique - est pris comme norme, la cyclicité du corps féminin constitue de fait une déviation de la norme (Kendall, 1991). La cyclicité devient alors instabilité - le corps féminin est (à nouveau) constitué en version instable, donc non fiable, incontrôlable et inférieure, du corps masculin.

\section{La pensée binaire}

\section{Le binarisme à l'épreuve des identités et des corps}

41 Dans les sociétés occidentales, le genre et le sexe sont généralement considérés comme binaires : il existe des femmes et des hommes, et tout individu est soit une femme soit un homme. Les femmes sont des femmes parce qu'elles ont un corps sexué femelle, avec un vagin, des ovaires, des chromosomes XX, etc. ; et les hommes sont des hommes parce qu'ils ont un corps sexué mâle, avec un pénis, des testicules, des chromosomes $\mathrm{XY}$, etc. Les différences entre les comportements féminins et masculins résulteraient par ailleurs des différences sexuées entre corps de femmes et corps d'hommes: les taux hormonaux, les structures cérébrales, les gènes, etc. Cette idéologie est largement partagée au sein de nombreuses sociétés et, comme l'ont montré des travaux comme ceux de Fausto-Sterling $(1987,2000)$ ou Kraus (2000), elle a aussi un effet configurant sur la construction des savoirs en sciences.

Cette bi-catégorisation des êtres humains en femme/féminine/femelle et homme/ masculin/mâle va cependant à l'encontre des ressentis, expériences et vécus d'un ensemble de personnes. Ces personnes, à travers leur non-conformité à ce schéma, montrent, d'une part, que des configurations différentes sont possibles, et d'autre part, que les subjectivités genrées et les anatomies attribuées par convention à des " sexes ${ }^{20}$ » féminins et masculins relèvent plus de continua que de variables binaires.

Pour illustrer que la bi-catégorisation des êtres humains en "femme » et «homme " résulte d'une idéologie de genre, différents auteur-e-s (comme p. ex. Bing \& Bergvall 1996 ; Bourcier 2001, 2005, 2011 ; Dvorsky \& Hughes 2008 ; Stryker 1998, etc.) mobilisent l'argument de l'existence d'identités autres, d'identités qui mettent à mal ce cadre binaire. Il existe p. ex. des personnes qui s'identifient comme genderqueer, trans queer, $\mathrm{Ft}^{*}$ (Female-to-Unknown), Mt* (Male-to-Unknown), genre fluide, intersexe, agenre, androgyne, tomboy, sissy, butch, folle, etc. Comme l'écrit Stryker (1998: 148), il y a une "wild profusion of gendered subject positions, spawned by the rupture of "woman" and "man" like an archipelago of identities rising from the sea ${ }^{21}$ ». Ces identités sont accomplies à travers des disjonctions du schéma que l'idéologie dominante présente comme normal : femme/féminine/femelle et homme/masculin/mâle. 
Avec les identités qui défont les conceptions binaires du genre, viennent aussi des corps qui défont les conceptions binaires du sexe. Ceci est notamment le cas des personnes trans et des personnes intersexes. Certaines personnes trans choisissent p. ex. de ne pas modifier chirurgicalement leurs organes génitaux et de modifier uniquement leurs caractères sexuels secondaires (p. ex. ablation des seins, implants mammaires, réduction de la pomme d'Adam, épilation, etc.), d'autres choisissent de les modifier seulement partiellement. Ces choix peuvent être motivés par différentes raisons: certaines personnes ne ressentent tout simplement pas le besoin de modifier leurs corps, d'autres n'ont pas la possibilité de le faire, et d'autres encore choisissent de ne pas se soumettre à des opérations lourdes dont les résultats sont parfois perçus comme peu satisfaisants (comme p. ex. la phalloplastie). Certaines personnes trans ont ainsi des corps qui ne correspondent pas aux conceptions des "corps normalement sexués ", c'est-à-dire un corps féminin avec un vagin, des seins, pas de pilosité faciale, etc. ; un corps masculin avec un pénis, une poitrine plate, etc.

D'autres corps qui remettent en question les conceptions binaires du sexe sont les corps des personnes intersexes (Blackless et al. 2000; Fausto-Sterling 1993, 2000). Les personnes intersexes ont des corps qui présentent des variations du développement sexuel (Picquart 2009: 123) qui provoquent des configurations génitales, gonadiques et chromosomiques n'obéissant pas au schéma vagin/ovaires/chromosomes-XX ou pénis/ testicules/chromosomes-XY. Les corps intersexes révèlent ainsi que la coprésence d'un vagin, d'ovaires et de chromosomes XX n'est pas systématique dans un corps assigné " femelle ", et que la coprésence d'un pénis, de testicules et de chromosomes XY ne l'est pas non plus dans un corps assigné «mâle", même si ces régularités peuvent être retrouvées dans une majeure partie des corps humains.

Selon la biologiste Fausto-Sterling (2000: 51), 1,7\% des enfants naissent intersexes. L'intersexualité n'est donc pas un phénomène si rare qu'il faudrait la disqualifier comme argument contre une vision binaire des sexes. La fréquence des naissances d'enfants intersexes a par ailleurs amené Fausto-Sterling à proposer une classification des êtres humains en cinq sexes : «male », « female », « merm », «ferm » et « herm » (1993). Elle faisait cette proposition « quelque peu ironique [...] pour introduire l'idée que notre sexe anatomique et physiologique implique bien plus que les organes génitaux, ce que l'on aperçoit en regardant les individus intersexués, parce que les différents niveaux du sexe (chromosomique, génital, hormonal, etc.) se trouvent en discordance » (Fausto-Sterling \& Touraille 2014).

Quelques années plus tard, Fausto-Sterling est revenue sur cette classification en cinq sexes pour introduire l'idée du «continuum sexuel». Elle explique les implications d'une conception du sexe en tant que continuum :

Si le mâle et la femelle se situent aux deux extrémités d'un continuum biologique, il existe bien d'autres corps [...] qui mêlent à l'évidence des éléments anatomiques attribués par convention aux hommes et aux femmes. Il y a de profondes implications à argumenter en faveur d'un continuum sexuel. Si la nature nous offre réellement davantage que deux sexes, il s'ensuit que nos notions actuelles de masculinité et de féminité sont des concepts culturels. Reconceptualiser la catégorie de "sexe", c'est remettre en cause l'un des éléments les plus solides de l'organisation sociale en Europe et en Amérique. (Fausto-Sterling 2012 [2000] : 52).

Ce que les corps intersexes révèlent également est que l'assignation d'un sexe à un corps n'est pas une décision qui se fonde uniquement sur des critères biologiques. Quand un enfant intersexe est né, une commission formée d'endocrinologues, de 
chirurgiens, de psychologues et de pédiatres va évaluer quel est le sexe de l'enfant. Étant donné que selon l'idéologie de genre dominante, l'enfant doit être ou bien de sexe féminin ou bien de sexe masculin, il faut «trouver s'il s'agit d'une fille ou d'un garçon et quel dérèglement, hormonal ou autre, a pu expliquer que ce vrai sexe soit masqué » (Picquart 2009 : 144). Les propos d'une endocrinologue rapportés par Picquart (id. : 144) illustrent parfaitement cela : " Il n'y a pas d'intersexes. Il n'y a que des pathologies et c'est aux médecins de déterminer si c'est un garçon ou une fille ». Ceci constitue l'argument généralement mobilisé par le corps médical pour intervenir sur les corps des enfants intersexes. Comme l'attestent grand nombre de témoignages (voir p. ex. Kessler 1998; Picquart 2009), les personnes intersexes subissent généralement plusieurs interventions dans leur enfance dans le but de normaliser leurs organes génitaux, c'est-à-dire leur donner l'apparence d'un vagin « acceptable » si les médecins ont décidé que l'enfant serait une fille, ou l'apparence d'un pénis «acceptable » si les médecins ont décidé que l'enfant serait un garçon. Comme l'indique Kessler (1998), cette acceptabilité repose souvent plus sur des critères esthétiques, donc sociaux, que sur des critères de fonctionnalité. Elle donne l'exemple des critères d'acceptabilité des longueurs de pénis et de clitoris (ibid. : 43) qui jouent dans la décision des médecins sur la manière dont il vont intervenir sur un organe jugé «ambigu »: en faire un pénis " acceptable" si l'organe est assez long ou bien le raccourcir ou amputer totalement pour en faire un clitoris « acceptable » si l'organe est trop petit.

Figure : Échelle d'acceptabilité des longueurs de pénis et clitoris, donnée par Kessler (1998: 43).

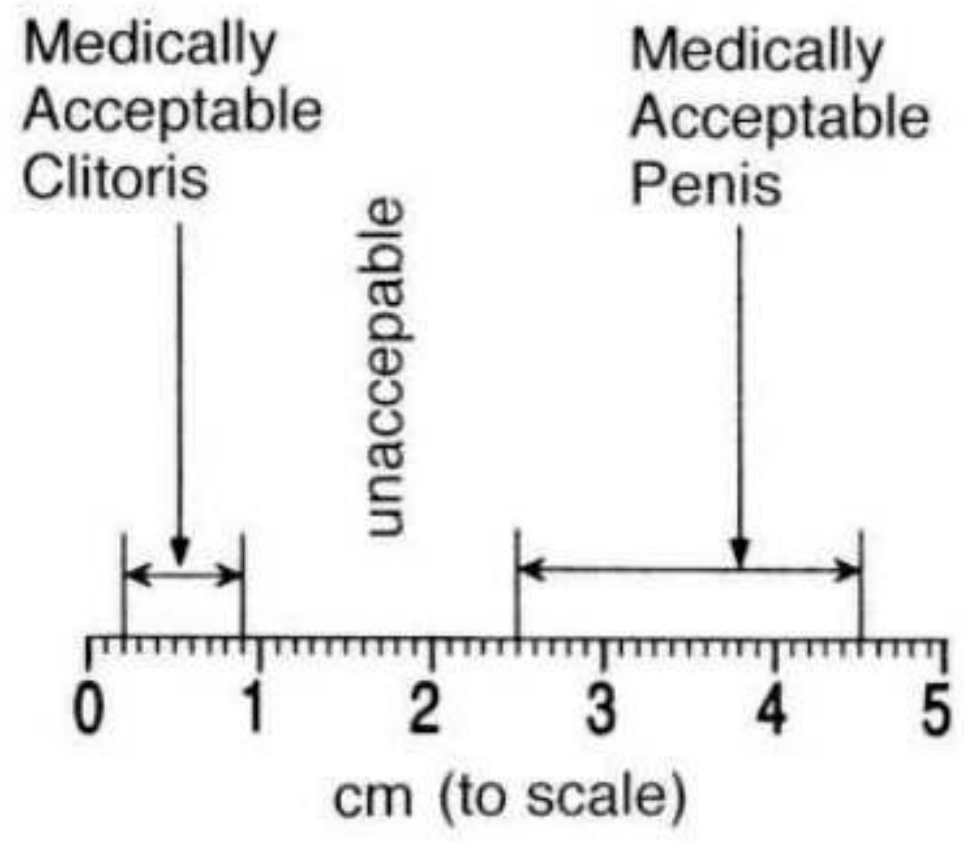

L'exemple des personnes trans et intersexes montre ainsi qu'il est impossible de classer les corps humains en deux et seulement deux classes homogènes en se basant sur des facteurs comme les organes génitaux, les gonades, les chromosomes ou les hormones. 


\section{Binarisme, attractivité et appareils phonatoires hybrides}

La littérature phonétique produit et reproduit l'idée du binarisme du genre et du sexe à travers les manières dont sont posées grand nombre de questions de recherche, formulées des hypothèses, ou encore élaborés des protocoles expérimentaux. J'étudierai dans cette section comment l'idéologie de la binarité est véhiculée par deux types d'études phonétiques: (1) les études portant sur l'attractivité des voix et (2) les études portant sur les variations morphologiques (présentées comme "anomalies») des appareils phonatoires à la suite de l'exposition à divers taux d'hormones. Le premier type d'étude véhicule l'idéologie de la binarité par la mobilisation du discours du «sexe/genre opposé» ou des discours selon lesquels l'attractivité s'exerce obligatoirement et uniquement entre femmes et hommes. Le deuxième type d'études véhicule l'idéologie de la binarité en mobilisant les discours du dimorphisme sexuel des appareils phonatoires et des discours pathologisant des appareils phonatoires présentés comme hybrides - c'est-à-dire composés d'éléments féminins et masculins.

51 L'analyse d'un échantillon de 24 études portant sur l'attractivité des voix (voir tableau 1) a permis d'établir différents constats. Dans un premier temps, j'ai remarqué que dans 12 études est utilisée l'expression « sexe opposé » ou " genre opposé ». En plus de constituer «femme » et " homme » comme identités tellement différentes qu'elles forment des opposés, cette expression renvoie à une vision du sexe/genre comme variable binaire : si «femme » est l'opposé de « homme », uniquement deux modalités sont possibles.

Dans un deuxième temps, j'ai constaté que dans 15 études est utilisé un protocole expérimental dans lequel l'attractivité des voix de femmes est uniquement évaluée par des hommes et l'attractivité des voix d'hommes est uniquement évaluée par des femmes. Seulement dans deux protocoles expérimentaux dans lesquels l'expression " sexe/genre opposé » n'apparaît pas, il est demandé à des évaluatrices/eurs de juger l'attractivité de voix de locutrices/eurs du même genre qu'elles/eux (Berry 1990, 1992).

Tableau : Études portant sur l'attractivité de voix

\begin{tabular}{|l|l|c|c|c|}
\hline & Étude & $\begin{array}{c}\text { Terme « sexe/ } \\
\text { genre opposé » }\end{array}$ & $\begin{array}{c}\text { Évaluation femme-homme, } \\
\text { homme-femme }\end{array}$ & $\begin{array}{c}\text { Aucun } \\
\text { des deux }\end{array}$ \\
\hline 1 & $\begin{array}{l}\text { Apicella \& Feinberg } \\
(2009)\end{array}$ & $\mathrm{x}$ & $\mathrm{x}$ & \\
\hline 2 & $\begin{array}{l}\text { Barkat-Defradas et al. } \\
(2012)\end{array}$ & & $\mathrm{x}$ & $\mathrm{x}$ \\
\hline 3 & $\begin{array}{r}\mathrm{x} \\
\text { Berry (1990) }\end{array}$ & $\mathrm{x}$ & $\mathrm{x}$ & \\
\hline 4 & Berry (1992) & $\begin{array}{l}\text { Borkowska \& Pawlowski } \\
(2011)\end{array}$ & & \\
\hline
\end{tabular}




\begin{tabular}{|c|c|c|c|c|}
\hline 6 & Brucker et al. (2006) & & $\mathrm{x}$ & \\
\hline 7 & Collins (2000) & & $\mathrm{x}$ & \\
\hline 8 & Collins \& Missing (2003) & & $\mathrm{x}$ & \\
\hline 9 & Feinberg et al. (2005) & $\mathrm{x}$ & $\mathrm{x}$ & \\
\hline 10 & Feinberg et al. (2006) & & $\mathrm{x}$ & \\
\hline 11 & Feinberg et al. (2008) & $\mathrm{x}$ & & \\
\hline 12 & Fraccaro et al. (2013) & $\mathrm{x}$ & & \\
\hline 13 & $\begin{array}{l}\text { Hodges-Simeon, Gaulin, } \\
\text { \& Puts (2010a) }\end{array}$ & $\mathrm{x}$ & $\mathrm{x}$ & \\
\hline 14 & $\begin{array}{l}\text { Hodges-Simeon, Gaulin, } \\
\text { \& Puts (2010b) }\end{array}$ & & $\mathrm{x}$ & \\
\hline 15 & $\begin{array}{l}\text { Hughes, Dispenza, \& } \\
\text { Gallup Jr. (2004) }\end{array}$ & $\mathrm{x}$ & & \\
\hline 16 & Jones et al. (2010) & $\mathrm{x}$ & & \\
\hline 17 & Lander (2008) & $\mathrm{x}$ & & \\
\hline 18 & Liu \& Xu (2011) & & $\mathrm{x}$ & \\
\hline 19 & $\begin{array}{l}\text { Pipitone \& Gallup Jr. } \\
(2008)\end{array}$ & $\mathrm{x}$ & & \\
\hline 20 & $\begin{array}{l}\text { Pisanski, Mishra \& } \\
\text { Rendall, (2012) }\end{array}$ & $\mathrm{x}$ & $x$ & \\
\hline 21 & Puts (2005) & & $\mathrm{x}$ & \\
\hline 22 & $\begin{array}{l}\text { Riding, Lonsdale \& } \\
\text { Brown (2006) }\end{array}$ & & $\mathrm{x}$ & \\
\hline 23 & Saxton et al. (2009) & $\mathrm{x}$ & & \\
\hline 24 & Vukovic et al. (2008) & & $\mathrm{x}$ & \\
\hline
\end{tabular}

Cette comparaison des protocoles expérimentaux permet de voir que, dans la littérature phonétique, il y a une tendance à concevoir l'attractivité comme un phénomène qui peut s'exercer uniquement entre membres appartenant à deux (et seulement deux) catégories de sexe/genre différentes: femmes et hommes. Elle véhicule ainsi un discours binariste.

Trois autres aspects rendent les études sur l'attractivité des voix problématiques. Le premier est que ces études véhiculent un discours sur la normalité du désir 
hétérosexuel. En présentant l'attractivité comme phénomène qui ne peut être évalué que par un membre du "sexe/genre opposé », ces études sous-entendent que le désir sexuel ne peut avoir lieu qu'entre membres du « sexe/genre opposé », c'est-à-dire entre femmes et hommes. L'hétérosexualité est ainsi présentée comme étant la sexualité normale, la sexualité qui va de soi. Si l'on pose l'hétérosexualité comme norme, toute autre sexualité devient spécifique, marquée, anormale. Il existe par ailleurs des études phonétiques qui, au-delà de réifier la figure de l'homosexuel-le, présentent les personnes homosexuelles comme étant potentiellement anatomiquement différentes des personnes hétérosexuelles. Par exemple Pierrehumbert et al. (2004:1905), dans une étude sur l'influence de l'orientation sexuelle sur la production des voyelles, posent l'hypothèse de départ suivante: "One possibility is that an innate biological factor influences both sexual orientation and the anatomical structures that underlie speech production $^{22}$. » Même si les auteur-e-s ne trouvent (évidemment) pas d'indice qui leur permette de corroborer cette hypothèse, le fait de la poser véhicule une vision de l'homosexuel comme essentiellement différent de l'hétérosexuel, qui est pris comme norme, considéré comme «normal ». Le deuxième aspect problématique des études sur l'attractivité des voix est qu'elles présentent l'attractivité comme biologique, universelle, et non pas comme socialement construite. Le fait que les canons de beauté et ainsi d'attractivité soient ancrés dans un espace-temps spécifique (voir p.ex. Reischer \& Koo 2004), qu'ils soient socialement situés, est complètement ignoré dans ces études. Le troisième aspect problématique est qu'elles confondent généralement attractivité et désir de reproduction. Ces études expliquent régulièrement que l'attractivité d'une voix est un indicateur de l'aptitude reproductrice du locuteur. Le raisonnement mobilisé présente souvent le schéma suivant: (1) Dans un premier temps, est réalisée une expérience perceptive qui montre que les évaluatrices/eurs présentent une préférence pour des voix stéréotypiques - sont préférées les voix de femmes aiguës ou/et claires et les voix d'homme graves ou/et sombres. (2) Ensuite est émis le postulat selon lequel cette préférence pour les voix stéréotypiques est d'origine biologique : l'influence de dynamiques sociales (canons de beauté) sur cette préférence n'est pas thématisée. (3) L'étape suivante est d'expliquer le lien entre hormones et voix féminine ou masculine. Par exemple, les hommes dont l'organisme présente un taux élevé de testostérone auraient des voix plus graves, etc. (4) Puis, est expliqué le lien entre hormones et aptitude d'un corps à la reproduction. (5) Finalement, la voix, parce qu'elle présente différentes caractéristiques en fonction des taux de testostérone, d'estrogènes ou de progestérone dans l'organisme, est présentée comme un indicateur de l'aptitude reproductrice d'un locuteur : les voix perçues comme attractives sont les voix des «bons reproducteurs", les voix perçues comme non attractives sont les voix des « moins bons reproducteurs".

Comme il a été expliqué plus haut, le discours binariste peut également être retrouvé dans les études portant sur les variations morphologiques des appareils phonatoires qui surviennent suite à l'exposition à divers taux d'hormones. Dans ces études, l'appareil phonatoire humain est régulièrement présenté comme sexuellement dimorphe : les plis vocaux (anciennement appelés " cordes vocales ») des femmes sont décrits comme étant moins longs, moins épais, moins massifs que ceux des hommes, et les conduits vocaux des femmes sont décrits comme étant moins longs, moins volumineux que ceux des hommes. Cependant, comme je l'avais déjà signalé autre part (Arnold 2016), il existe une grande variabilité dans les tailles des plis vocaux et des conduits vocaux : certaines femmes ont des plis vocaux et des conduits vocaux plus 
longs que certains hommes, et certains hommes ont des plis vocaux et conduits vocaux plus courts que certaines femmes. À partir de ce constat, l'idée du dimorphisme sexuel des appareils phonatoires devient problématique. Pour Fausto-Sterling (Fausto-Sterling \& Touraille 2014: §2), «quand on parle de différences sexuées [...] des caractères sexuels secondaires, le concept de continuum est plus pertinent [que celui de dimorphisme]. [...] [C]es traits qui présentent des différences collectives moyennes entre populations issues d'aires géographiques similaires ne sont clairement pas dimorphes. [...] Ce sont des moyennes relatives au sexe pour des traits qui sont distribués de manière continue ». On peut ainsi s'apercevoir qu'il existe une confusion entre moyennes et tendances que l'on retrouve dans les corps assignés "femelle » et «mâle ».

Malgré la grande variabilité qui existe dans les appareils phonatoires féminins et masculins, la littérature phonétique les qualifie régulièrement de dimorphes et véhicule un discours normatif sur les tailles et dimensions jugées acceptables et inacceptables pour les femmes et les hommes. Quand certains taux hormonaux provoquent des formes laryngées atypiques, c'est-à-dire des plis vocaux jugés anormalement volumineux pour des femmes, ou jugés anormalement petits pour des hommes, ceux-ci sont décrits par les termes de "androglottia » et "gynecoglottia » (Azul 2013: 84). Le terme "gynecoglottia» désigne des plis vocaux «de forme féminine " chez les hommes, et le terme "androglottia " des plis vocaux «de forme masculine » chez les femmes (Hirschfeld 1910 [2006:36]). L'androglottia provoque une voix relativement grave et la gynecoglottia, une voix relativement aiguë.

57 Le fait que des formes laryngées soient désignées par les termes "gynecoglottia » et "androglottia" sous-entend qu'il existe des formes laryngées propres aux corps féminins et d'autres propres aux corps masculins. Des études qui mobilisent ces termes constituent implicitement les corps des personnes étudiées en corps hybrides, des corps qui mélangent ce qui est propre à la femme et ce qui est propre à l'homme. Ces corps hybrides (du grec "hybris»: démesure) évoquent ainsi l'anormalité, la monstruosité que constitue la non-concordance au schéma binaire.

On voit un discours similaire mobilisé dans les publications portant sur les changements laryngés et vocaux qui surviennent chez des femmes ménopausées à la suite de modifications des taux de testostérone, d'estrogènes et de progestérone dans l'organisme. Dans ces publications les termes « masculinisation » et « virilisation » sont régulièrement utilisés pour désigner l'épaississement des plis vocaux et les changements de voix qui peuvent en résulter. En décrivant des larynx de femmes comme masculinisés, virilisés, le discours du corps hybride cité plus haut est à nouveau mobilisé. Ce discours perpétue ainsi la vision de la binarité des corps normalement sexués. Une similitude entre les processus de pathologisation des changements qui surviennent après la ménopause et les changements qui surviennent au cours du cycle menstruel (voir section 2) peut par ailleurs être constatée: ces deux changements surviennent régulièrement dans les corps de nombreuses femmes, mais sont présentés comme des anomalies ou pathologies. 


\section{Conclusions}

59 Ces différents exemples ont permis d'exposer comment les savoirs produits dans les sciences phonétiques sont, malgré leur objectivité et neutralité axiologique postulées, empreints d'idéologies et contribuent à véhiculer celles-ci.

- Une analyse de la littérature phonétique a permis de voir que le corps masculin est systématiquement utilisé pour représenter l'être humain. Le corps masculin est ainsi érigé en norme et le corps féminin devient spécifique et marqué.

- Une des conséquences de cette normalisation du corps masculin est que le corps féminin et sa cyclicité sont pathologisés.

- L'analyse d'études portant sur l'attractivité des voix et sur des variations morphologiques des appareils phonatoires (présentées comme "anomalies ») a ensuite permis de montrer que la littérature phonétique véhicule une idéologie de genre binaire qui normalise les schémas femme/féminine/femelle et homme/masculin/mâle.

À l'instar d'autres domaines scientifiques, les sciences phonétiques gagneraient à abandonner la position confortable du positivisme et à commencer à questionner la dimension idéologique et genrée de la production des savoirs, ainsi que la notion même d'objectivité. Comme l'explique Haraway (1988), l'abandon du positivisme n'implique pas d'adopter obligatoirement une vision constructiviste radicale qui pourrait pousser à nier la nécessité même d'un processus de recherche. En revanche, concevoir l'objectivité comme incarnée et les savoirs scientifiques comme situés et partiels permettrait de déjouer certains biais sexistes et binaristes que l'on retrouve à ce jour encore bien trop fréquemment en phonétique.

\section{BIBLIOGRAPHIE}

ARNOLD, Aron. 2016. « Voix », in Encyclopédie critique du genre, RENNES, Juliette et al. (éd.). Paris : La Découverte, 713-721.

ABITBOL, Jean. 2013 [2005]. L'odyssée de la voix. Paris: Flammarion.

ABITBOL, Jean, ABITBOL, Patrick \& ABITBOL, Béatrice. 1999. « Sex hormones and the female voice » Journal of Voice 13: 424-446.

ABITBOL, Jean, DE BRUX, Jean, MILLOT, Ginette, MASSON, Marie-Francoise, LANGUILLE MIMOUN, Odile, PAU, Helene \& ABITBOL, Beatrice. 1989. « Does a hormonal vocal cord cycle exist in women? Study of vocal premenstrual syndrome in voice performers by videostroboscopyglottography and cytology on 38 women » Journal of Voice 3: 157-162.

APICELLA, Coren L. \& FEINBERG, David R. 2009. « Voice pitch alters mate-choice-relevant perception in hunter-gatherers » Proceedings of the Royal Society B: Biological Sciences 276: 1077-1082.

AZUL, David. 2013. « How Do Voices Become Gendered? A Critical Examination of Everyday and Medical Constructions of the Relationship Between Voice, Sex, and Gender Identity », in 
Challenging Popular Myths of Sex, Gender and Biology, Crossroads of Knowledge 1, AH-KING, Malin (éd.). New York: Springer International Publishing, 77-88.

BARKAT-DEFRADAS, Melissa, BUSSEUIL, Coralie, CHAUVY, Oriane, HIRSCH, Fabrice, FAUTH, Camille, REVIS, Joana \& AMY DE LA BRETĖQUE, Benoît. 2012. « Dimension esthétique des voix normales et dysphoniques : Approches perceptive et acoustique » TIPA. Travaux interdisciplinaires sur la parole et le langage 28.

BEHR DAVIS, Clarissa \& LEE DAVIS, Michael. 1993. « The effects of premenstrual syndrome (PMS) on the female singer » Journal of Voice 7: 337-353.

BERRY, Diane S. 1990. « Vocal attractiveness and vocal babyishness: Effects on stranger, self, and friend impressions » Journal of Nonverbal Behavior 14:141-153.

BERRY, Diane S.,1992. « Vocal types and stereotypes: Joint effects of vocal attractiveness and vocal maturity on person perception » Journal of Nonverbal Behavior 16: 41-54.

BING, Janet M. \& BERGVALL, Victoria L. 1996. «The Question of Questions: Beyond Binary Thinking ", in Rethinking language and gender research: theory and practice, BERGVALL, Victoria L., BING, Janet M., FREED, Alice F. (éd.). Harlow: Longman, 1-30.

BLACKLESS, Melanie, CHARUVASTRA, Anthony, DERRYCK, Amanda, FAUSTO-STERLING, Anne, LAUZANNE, Karl \& LEE, Ellen. 2000. « How sexually dimorphic are we? Review and synthesis » American Journal of Human Biology: The Official Journal of the Human Biology Council 12: 151-166.

BOË, Louis-Jean, HEIM, Jean-Louis, HONDA, Kiyoshi, MAEDA, Shinji, BADIN, Pierre \& ABRY, Christian. 2007. «The vocal tract of newborn humans and Neanderthals: Acoustic capabilities and consequences for the debate on the origin of language. A reply to Lieberman » Journal of Phonetics 35: 564-581.

BORKOWSKA, Barbara \& PAWLOWSKI, Boguslaw. 2011. «Female voice frequency in the context of dominance and attractiveness perception » Animal Behaviour 82: 55-59.

BOURCIER, Marie-Hélène. 2001. Queer zones : politiques des identités sexuelles, des représentations et des savoirs. Paris : Balland.

BOURCIER, Marie-Hélène. 2005. Sexpolitiques : queer zones 2. Paris : La Fabrique.

BOURCIER, Marie-Hélène. 2011. Queer Zones 3, Identités, cultures et politiques. Paris : Éditions Amsterdam.

BOURCIER, Marie-Hélène \& MOLINER Alice. 2012. Comprendre le féminisme. Paris : Max Milo.

CALLIOPE. 1989. La Parole et son traitement automatique. Paris: Masson \& CNET-ENST.

CAMPBELL, Joseph P. 1997. « Speaker recognition: a tutorial » Proceedings of the IEEE 85: 1437-1462.

COLLINS, Sarah A. 2000. « Men's voices and women's choices » Animal Behaviour 60: 773-780.

COLLINS, Sarah A. \& MISSING, Caroline. 2003. « Vocal and visual attractiveness are related in women » Animal Behaviour 65: 997-1004.

DELATTRE, Pierre. 1968. « La Radiographie des voyelles françaises et sa correlation acoustique » The French Review 42 : 48-65.

DELLWO, Volker, HUCKVALE, Mark \& ASHBY, Michael. 2007. « His is Individuality Expressed in Voice? An Introduction to Speech Production and Description for Speaker Classification », in Speaker Classification I: Fundamentals, Features, and Methods, MÜLLER, Christian (éd.). New York : Springer International Publishing, 1-20. 
DORLIN, Elsa. 2005. « Les Blanchisseuses. La société plantocratique antillaise, laboratoire de la féminité moderne ", in Le corps, entre sexe et genre, ROUCH, Hélène, DORLIN, Elsa \& FOUGEYROLLAS-SCHWEBEL, Dominique (éd.). Paris : L'Harmattan, 129-148.

DORLIN, Elsa. 2006. La matrice de la race : Généalogie sexuelle et coloniale de la nation française. Paris: La Découverte.

DVORSKY, George \& HUGHES, James. 2008. « Postgenderism: Beyond the gender binary » IEET White Paper Series 3, 1-18.

ECKERT, Penelope \& MCCONNELL-GINET, Sally. 2003. Language and Gender. Cambridge: Cambridge University Press.

FANT, Gunnar. 1960. Acoustic theory of speech production: with calculations based on X-ray studies of Russian articulations. The Hague: Mouton \& Co.

FANT Gunnar, 1966, «A note on vocal tract size factors and non-uniform F-pattern scalings ", STL-QPSR, 7: 022-030.

FANT, Gunnar. 2004. « Speech research in a historical perspective ». Proceedings of From sound to sense: 50+ years of discoveries in speech communication. Research Laboratory of Electronics [En ligne], consulté le 30 mai 2016. URL: http://www.rle.mit.edu/soundtosense/conference/pdfs/ invitedspeakers/Fant\%20PAPER.pdf

FAUSTO-STERLING, Anne. 1987. Myths of Gender: Biological Theories about Women and Men. New York: Basic Books.

FAUSTO-STERLING, Anne. 1993. « The Five Sexes - Why Male and Female are not Enough » The Sciences 33: 19-25.

FAUSTO-STERLING, Anne. 2012 [2000]. Corps en tous genres. Paris : La Découverte.

FAUSTO-STERLING, Anne \& TOURAILLE, Priscille. 2014. « Autour des critiques du concept de sexe. Entretien avec Anne Fausto-Sterling » Genre, sexualité \& société 12.

FEINBERG, David R., DEBRUINE, Lisa M., JONES, Benedict C. \& LITTLE, Anthony C. 2008. «Correlated preferences for men's facial and vocal masculinity » Evolution and Human Behavior 29: 233-241.

FEINBERG, David R., JONES, Benedict C., LAW SMITH, Miriam J., MOORE, Fhionna R., DEBRUINE, Lisa M., CORNWELL, Robin E., HILLIER, Stephen, G. \& PERRETT, David I. 2006. « Menstrual cycle, trait estrogen level, and masculinity preferences in the human voice » Hormones and Behavior 49: 215-222.

FEINBERG, David R., JONES, Benedict C., LITTLE, Anthony C., BURT, David M. \& PERRETT, David I. 2005. « Manipulations of fundamental and formant frequencies influence the attractiveness of human male voices » Animal Behaviour 69: 561-568.

FRABLE, Mary Ann. 1962. « Hoarseness, a symptom of premenstrual tension » Archives of otolaryngology 75: 66-68.

FRACCARO, Paul J. \& O'CONNOR, Jillian J. M., RE, Daniel E., JONES, Benedict C., DEBRUINE, Lisa M. \& FEINBERG, David R. 2013. «Faking it: deliberately altered voice pitch and vocal attractiveness » Animal Behaviour 85: 127-136.

FRANK, Robert T. 1931. « The hormonal causes of premenstrual tension » Archives of Neurology \& Psychiatry 26: 1053-1057. 
GRECO, Luca. 2013. «Exhumer le corps du placard. Pour une linguistique queer du corps king », in Ecritures du corps - Nouvelles perspectives, ZOBERMAN, Pierre, TOMICHE, Anne \& SPURLIN, William (éd.). Paris: Classiques Garnier, 269-289.

GREENE, Raymond \& DALTON, Katharina. 1953. « The Premenstrual Syndrome » British Medical Journal 1: 1007-1014.

GUILLAUMIN, Colette. 1978. « Pratique du pouvoir et idée de Nature (2) Le discours de la Nature » Questions Féministes 3 : 5-28.

HARAWAY, Donna. 1988. « Situated Knowledges: The Science Question in Feminism and the Privilege of Partial Perspective » Feminist Studies 14: 575-599.

HENTON, Caroline Gilles. 1986. A comparative study of phonetic sex-specific differences across langages. Thèse de doctorat. Oxford : University of Oxford.

HERITIER, Françoise. 1984. « Le sang du guerrier et le sang des femmes » Les Cahiers du GRIF 29 : 7-21.

HIGGINS, Maureen B. \& SAXMAN, John H. 1989. « Variations in vocal frequency perturbation across the menstrual cycle » Journal of Voice 3: 233-243.

HIRSCHFELD Magnus, (1910) 2006, « Selections from The Transvestites: The Erotic Drive to CrossDress ", in STRYKER Susan, WHITTLE Stephen (éd.), The transgender studies reader, New York: Routledge, 28-39

HODGES-SIMEON, Carolyn R., GAULIN, Steven J. C. \& PUTS, David A. 2010a. « Voice Correlates of Mating Success in Men: Examining “Contests" Versus "Mate Choice" Modes of Sexual Selection » Archives of Sexual Behavior 40(3): 551-557.

HODGES-SIMEON, Carolyn R., GAULIN, Steven J. C. \& PUTS, David A. 2010b. « Different Vocal Parameters Predict Perceptions of Dominance and Attractiveness » Human Nature 21: 406-427.

HUGHES, Susan M., DISPENZA, Franco \& GALLUP, Gordon G. 2004. « Ratings of voice attractiveness predict sexual behavior and body configuration » Evolution and Human Behavior 25: 295-304.

JAKOBSON, Roman, FANT, Gunnar \& HALLE, Morris. 1963. Preliminaries to speech analysis: the distinctive features and their correlates. Cambridge: MIT Press.

JOHNSON, Thomas M. 1987. «Premenstrual syndrome as a Western culture-specific disorder » Culture, Medicine and Psychiatry 11: 337-356.

JONES, Benedict C., FEINBERG, David R., DEBRUINE, Lisa M., LITTLE, Anthony C. \& VUKOVIC, Jovana. 2010. « A domain-specific opposite-sex bias in human preferences for manipulated voice pitch » Animal Behaviour 79: 57-62.

KENDALL, Kathy. 1991. « The politics of Premenstrual Syndrome: Implications for feminist justice » Critical Criminology 2: 77-98.

KESSLER, Suzanne J. 1998. Lessons from the intersexed. Brunswick: Rutgers University Press.

KRAUS, Cynthia. 2000. "La bicatégorisation par sexe à l'“épreuve de la science" ", in L'Invention du naturel : Les sciences et la fabrication du féminin et du masculin, GARDEY, Delphine \& LOWY, Ilana (éd.). Paris: Éditions des archives contemporaines, 187-213.

LADEFOGED, Peter. 1996. Elements of Acoustic Phonetics. Chicago: University of Chicago Press.

LANDER, Karen. 2008. « Relating visual and vocal attractiveness for moving and static faces » Animal Behaviour 75: 817-822. 
LANDERCY, Albert \& RENARD, Raymond. 1979. Éléments de phonétique. Paris: Didier.

LAQUEUR, Thomas W. 1990. Making sex: Body and Gender from the Greeks to Freud. Cambridge: Harvard University Press.

LAWRENCE, Susan C. \& BENDIXEN, Kae. 1992. « His and hers: male and female anatomy in anatomy texts for U.S. medical students, 1890-1989 » Social Science \& Medicine 35: 925-934.

LIEBERMAN P., 1986, « Some aspects of dimorphism and human speech », Human Evolution, 1: 6775.

LIU, Xuan \& XU, Yi. 2011. « What Makes Female Voice Attractive ». Proceedings of ICPhS XVII, 1274-1277.

MARTIN, Philippe. 2008. Phonétique acoustique : Introduction à l'analyse acoustique de la parole. Paris : Armand Colin.

MEURER, Elisea M., GARCEZ, Vera, VON EYE CORLETA, Helena \& CAPP, Edison. 2009. « Menstrual Cycle Influences on Voice and Speech in Adolescent Females » Journal of Voice 23 : 109-113.

MICHARD, Claire. 2000. « Sexe et humanité en français contemporain » L'Homme. Revue française d'anthropologie 153 : 125-152.

MIRANDA, Eduardo. 2012. Computer Sound Design : Synthesis techniques and programming. Abingdon: Focal Press.

MORRIS, Richard J., McCREA, Christopher R. \& HERRING, Kaileen D. 2008. « Voice onset time differences between adult males and females: Isolated syllables » Journal of Phonetics 36: 308-317.

ORMEZZANO, Yves. 2000. Le guide de la voix. Paris : Odile Jacob.

PICQUART, Julien. 2009. Ni homme, ni femme : enquête sur l'intersexuation. Paris : La Musardine.

PIERREHUMBERT, Janet B., BENT, Tessa, MUNSON, Benjamin, BRADLOW, Ann R. \& BAILEY, Michael. 2004. "The influence of sexual orientation on vowel production » The Journal of the Acoustical Society of America 116(4): 1905-1908.

PIPITONE, Nathan \& GALLUP, Gordon G. 2008. « Women's voice attractiveness varies across the menstrual cycle » Evolution and Human Behavior 29: 268-274.

PISANSKI, Katarzyna, MISHRA, Sandeep \& RENDALL, Drew. 2012. « The evolved psychology of voice: evaluating interrelationships in listeners' assessments of the size, masculinity, and attractiveness of unseen speakers » Evolution and Human Behavior 33: 509-519.

PUTS, David A. 2005. « Mating context and menstrual phase affect women's preferences for male voice pitch » Evolution and Human Behavior 26: 388-397.

REISCHER, Erica \& KOO, Kathryn S. 2004. «The Body Beautiful: Symbolism and Agency in the Social World » Annual Review of Anthropology 33: 297-317.

RIDING, David, LONSDALE, Deryle \& BROWN, Bruce. 2006. « The Effects of Average Fundamental Frequency and Variance of Fundamental Frequency on Male Vocal Attractiveness to Women » Journal of Nonverbal Behavior 30: 55-61.

RODIN, Mari. 1992. « The social construction of premenstrual syndrome » Social Science \& Medicine 35: 49-56.

SAXTON, Tamsin K., DEBRUINE, Lisa M., JONES, Benedict C., LITTLE, Anthony C. \& ROBERTS, S. Craig. 2009. «Face and voice attractiveness judgments change during adolescence » Evolution and Human Behavior 30: 398-408. 
SCHROEDER, Manfred R. 2013. Computer Speech: Recognition, Compression, Synthesis. New York: Springer Science \& Business Media.

SILVERMAN, Ellen-Marie \& ZIMMER, Catherine H. 1978. « Effect of the menstrual cycle on voice quality » Archives of Otolaryngology 104: 7-10.

STRYKER, Susan. 1998. « The Transgender Issue: An Introduction » GLQ: A Journal of Lesbian and Gay Studies 4: 145-158.

TITZE, Ingo R. 2001. « Acoustic Interpretation of Resonant Voice » Journal of Voice 15: 519-528.

VUKOVIC, Jovana, FEINBERG, David R., JONES, Benedict C., DEBRUINE, Lisa M., WELLING, Lisa L. M., LITTLE, Anthony C. \& SMITH, Finlay G. 2008. « Self-rated attractiveness predicts individual differences in women's preferences for masculine men's voices » Personality and Individual Differences 45: 451-456.

WITTIG, Monique. 1980. « La pensée straight » Questions Féministes 7 : 45-53.

ZITA, Jacquelyn N. 1988. « The Premenstrual Syndrome "Dis-easing” the Female Cycle » Hypatia 3: 77-99.

\section{NOTES}

1. «l'ensemble des croyances qui gouvernent la participation des individus à l'ordre du genre, et par lesquelles ils expliquent et justifient cette participation » (ma traduction).

2. Wittig (1980: 50) rappelle que le concept de «différence des sexes» constitue « ontologiquement les femmes en autres différents. Les hommes eux ne sont pas différents. (Les Blancs non plus d'ailleurs ni les maîtres mais les Noirs le sont et les esclaves aussi) ».

3. «Choisir des illustrations de corps masculins pour représenter des caractéristiques nonsexuées du corps, organiser des chapitres en sections sur telle ou telle partie du corps humain distinctes des sections portant sur "la femme", comparer explicitement ou implicitement les structures féminines aux structures masculines, mettre des termes féminins entre parenthèses, et présenter les anatomies féminines comme des parties masculines altérées - tout cela participe au maintien d'une hiérarchie des anatomies : le masculin, puis le féminin; le masculin comme norme, le féminin comme différent » (ma traduction).

4. https://www.skidmore.edu/ hfoley/Perc11.htm

5. Ainsi, avec un conduit vocal moyen deux fois moins long $(8 \mathrm{~cm})$ que celui d'un humain adulte $(17 \mathrm{~cm})$, un nourrisson âgé de quelques mois produit des voyelles dont les formants sont deux fois plus élevés que ceux d'un adulte (ma traduction).

6. [...] ses fréquences de résonance étant visiblement plus basses que celles produites par un conduit vocal normal d'une longueur de $170 \mathrm{~mm}$ » chez les femmes (Hirschfeld,M(ma traduction).

7. La figure 3 montre l'inertance du conduit vocal en tant que fonction de la fréquence fondamentale pour un conduit d'une longueur de $17.5 \mathrm{~cm}$ complètement ouvert au niveau de la bouche (ma traduction).

8. « Nous considérerons le conduit vocal comme un tube d'environ $17.5 \mathrm{~cm}$ de long (il est un peu moins long chez la plupart des locuteurs, mais utiliser ce nombre est pratique parce qu'il simplifie les calculs)» (ma traduction).

9. Un conduit vocal d'un homme adulte a une longueur approximative de $17 \mathrm{~cm}$. (ma traduction) 10. Étant donné que la longueur moyenne du conduit vocal d'un homme a environ $17.5 \mathrm{~cm}$, les résonances se situent approximativement à 500, 1500, 2500 cps, etc. (ma traduction). 
11. Ceci explique pourquoi les fréquences de formants des femmes adultes sont en moyenne plus élevées que celles des hommes, étant donné qu'un conduit vocal de femme adulte typique est plus court que celui d'un homme adulte (ma traduction).

12. Un conduit vocal de femme typique est 15 à 20 pourcents plus court que celui d'un homme typique (ma traduction).

13. Certaines hypothèses sur les différences de VOT entre hommes et femmes ont été formulées en se basant sur le fait que les volumes des conduits vocaux sont plus petits chez les femmes (ma traduction).

14. " pendant deux millénaires, il n'existait même pas de terme spécifique pour désigner l'ovaire, alors que cet organe est devenu au début du dix-neuvième siècle une synecdoque pour femme » (ma traduction).

15. Le terme « hystérie " vient du mot grec « hystera » qui signifie « utérus ».

16. Le Diagnostic and Statistical Manual of Mental Disorders (DSM) est un manuel de référence publié par la American Psychiatric Association. Il classifie et catégorise des troubles mentaux et leurs critères diagnostiques.

17. Chez 38 femmes, nous avons trouvé 22 syndromes vocaux prémenstruels, se manifestant par un enrouement de voix et une augmentation de fatigue vocale. Des frottis des plis vocaux ont confirmé que ces 22 femmes souffraient toutes d'insuffisance lutéale. Seize femmes ne présentaient pas de modification de la voix particulière pendant la phase prémenstruelle, bien que deux d'entre elles aient une insuffisance lutéale. Quatorze avaient une sécrétion hormonale normale (ma traduction).

18. Le syndrome vocal prémenstruel se caractérise par une fatigue vocale, un registre réduit, une perte de force et une perte de certains harmoniques. Le syndrome commence habituellement 4-5 jours avant la menstruation chez environ $33 \%$ des femmes. Les professionnelles de la voix sont particulièrement touchées. Une exploration vocale dynamique par télévidéoendoscopie montre des congestions, des micro-varices, des œdèmes du tiers postérieur des plis vocaux ainsi qu'une perte d'amplitude vibratoire (ma traduction).

19. L'objectif de cet article est de présenter l'enrouement comme un symptôme méconnu de la tension prémenstruelle, de décrire brièvement cette entité, de discuter des mécanismes responsables de la raucité vocale et de la perte du contrôle vocal, et de donner des exemples cliniques. (ma traduction)

20. Le terme " sexe " est utilisé ici au sens d'une construction sociale mobilisant la fiction d'un fondement biologique permettant une différenciation binaire des corps en «femelle » et « mâle ».

21. "profusion folle de subjectivités genrées, engendrée par la rupture de "femme" et "homme", se soulevant comme un archipel d'identités de la mer » (ma traduction).

22. «Une possibilité est que ce soit un facteur biologique inné qui influence à la fois l'orientation sexuelle et le développement des structures anatomiques sollicitées lors de la production de la parole » (ma traduction).

\section{RÉSUMÉS}

L'objectif de cet article est d'exposer comment les savoirs produits dans les sciences phonétiques sont, malgré leur objectivité et neutralité axiologique postulées, empreintes d'idéologies de genre et contribuent à véhiculer celles-ci. Dans la littérature phonétique, le corps est 
omniprésent. Quand la production d'un son est décrite, le corps du locuteur est régulièrement représenté par un corps masculin. Par exemple, le conduit vocal humain est régulièrement présenté comme ayant une longueur de $17 / 17,5 \mathrm{~cm}$. Cette longueur ne correspond cependant pas à une moyenne des conduits vocaux humains, mais à une moyenne de conduits vocaux masculins. Une des conséquences de la confusion entre humain et masculin est que le corps masculin est instauré en norme, et que le corps féminin, présenté comme spécifique, marqué, peut faire l'objet d'un discours pathologisant. Ceci est notamment le cas dans la littérature sur le Syndrome vocal prémenstruel, où des phénomènes physiologiques accompagnant la cyclicité féminine sont décrits en tant que "symptômes " d'un « syndrome ». Le troisième discours idéologique étudié est celui du binarisme. La littérature phonétique produit et reproduit l'idée du binarisme de genre à travers les manières dont sont posées des questions de recherche, formulées des hypothèses, ou encore élaborés des protocoles expérimentaux. Par exemple, dans des textes phonétiques, l'expression «sexe/genre opposé » apparaît régulièrement et l'appareil vocal humain est systématiquement décrit comme sexuellement dimorphe.

This article exposes how knowledge construction in phonetic sciences is influenced by gender ideologies. The idea that the production of knowledge is a neutral act is deconstructed and it is instead demonstrated that scientific knowledge is situated knowledge and thus shaped by specific assumptions about gender. In phonetic literature, the body is omnipresent. In descriptions of how human sounds are produced, the body of the speaker is frequently represented by a male body. For example, the human vocal tract is frequently defined as being 17 or $17,5 \mathrm{~cm}$ long. This is not the average length of human vocal tracts but the average length of male vocal tracts as measured in early studies. As a consequence of the confusion between human and male, the male body becomes the norm, and the female body becomes specific, marked, and eventually pathologized. This is the case, for instance, in the literature about Premenstrual Vocal Syndrome, where different physiological phenomena accompanying the female cyclicity are being described as "symptoms" of a "syndrome". Gender binarism is the third ideology we'll analyse. Phonetic literature produces the idea of gender binary in the very way research questions and hypotheses are formulated and experimental protocols designed. For example, in phonetic texts, the expression "opposite gender/sex" appears frequently, and the human vocal apparatus is systematically described as sexually dimorphic.

\section{INDEX}

Mots-clés : épistémologie féministe, genre, idéologie, phonétique, savoirs scientifiques

Thèmes : Recherches

Keywords : feminist epistemology, gender, ideology, phonetics, scientific knowledges

\section{AUTEUR}

\section{ARON ARNOLD}

Laboratoire de phonétique et phonologie

UMR 7018 / CNRS \& Université Sorbonne nouvelle 